\title{
Ingestion of coffee polyphenols increases postprandial release of the active glucagon-like peptide-1 (GLP-1(7-36)) amide in C57BL/6J mice
}

\author{
Yoshie Fujii, Noriko Osaki, Tadashi Hase and Akira Shimotoyodome* \\ Biological Science Laboratories, Kao Corporation, Tochigi, Japan \\ (Received 29 June 2014 - Final revision received 30 July 2014 - Accepted 14 November 2014) \\ Journal of Nutritional Science (2015), vol. 4, e9, page 1 of 9
}

doi:10.1017/jns.2014.71

Abstract

The widespread prevalence of diabetes, caused by impaired insulin secretion and insulin resistance, is now a worldwide health problem. Glucagon-like peptide 1 (GLP-1) is a major intestinal hormone that stimulates glucose-induced insulin secretion from $\beta$ cells. Prolonged activation of the GLP-1 signal has been shown to attenuate diabetes in animals and human subjects. Therefore, GLP-1 secretagogues are attractive targets for the treatment of diabetes. Recent epidemiological studies have reported that an increase in daily coffee consumption lowers diabetes risk. The present study examined the hypothesis that the reduction in diabetes risk associated with coffee consumption may be mediated by the stimulation of GLP-1 release by coffee polyphenol extract (CPE). GLP-1 secretion by human enteroendocrine NCI-H716 cells was augmented in a dose-dependent manner by the addition of CPE, and was compatible with the increase in observed active GLP-1(7-36) amide levels in the portal blood after administration with CPE alone in mice. CPE increased intracellular cyclic AMP (cAMP) levels in a dose-dependent manner, but this was not mediated by G protein-coupled receptor 119 (GPR119). The oral administration of CPE increased diet (starch and glyceryl trioleate)-induced active GLP-1 secretion and decreased glucose-dependent insulinotropic polypeptide release. Although CPE administration did not affect diet-induced insulin secretion, it decreased postprandial hyperglycaemia, which indicates that higher GLP-1 levels after the ingestion of CPE may improve insulin sensitivity. We conclude that dietary coffee polyphenols augment gut-derived active GLP-1 secretion via the cAMP-dependent pathway, which may contribute to the reduced risk of type 2 diabetes associated with daily coffee consumption.

Key words: Blood glucose: Cyclic AMP: Glucose-dependent insulinotropic polypeptide: Glucagon-like peptide-1: NCI-H716

The widespread prevalence of diabetes, caused by impaired insulin secretion and insulin resistance, is now a worldwide health problem. Global statistics estimated that there were 382 million individuals with diabetes worldwide in 2014, and the number of individuals who are affected with diabetes will reach over 592 million by $2035^{(1)}$.

Glucagon-like peptide-1 (GLP-1) is a gut-derived hormone that stimulates the glucose-induced secretion of insulin from pancreatic $\beta$-cells. Prolonged activation of the GLP-1 signal has been shown to attenuate diabetes in animals and human subjects. GLP-1 secretagogues could represent a practicable option for patients to control their blood glucose levels ${ }^{(2)}$. Whereas dietary supplementation with GLP-1 stimulators could offer potential benefits for the treatment of diabetes, food-derived GLP-1 modulators have not yet been examined in detail.

Coffee is one of the most popular beverages throughout the world and has been consumed for thousands of years due to its attractive flavour and physiological effects. Many studies have demonstrated a relationship between the consumption of coffee and potential health benefits. Liver cirrhosis ${ }^{(3)}$, liver and colorectal cancer ${ }^{(4,5)}$ and death due to $\mathrm{CVD}^{(6)}$ have Abbreviations: BW, body weight; cAMP, cyclic AMP; CHO-K1, Chinese hamster ovary-K1; CPE, coffee polyphenol extract; CQA, caffeolylquinic acid; DPP IV, dipeptidyl
peptidase IV; FBS, fetal bovine serum; FQA, feruloylquinic acid; GIP, glucose-dependent insulinotropic polypeptide; GLP-1, glucagon-like peptide-1; GPR119, G proteincoupled receptor 119; TO, glyceryl trioleate.

* Corresponding author: Dr Akira Shimotoyodome, fax +81 28568 7495, email shimotoyodome.akira@kao.co.jp 
previously been shown to be reduced by the consumption of coffee. Recent epidemiological studies reported that an increase in the daily consumption of coffee was associated with a reduced risk of diabetes ${ }^{(7)}$, and similar findings have been found for decaffeinated coffee consumption ${ }^{(8)}$. However, the components in coffee that are responsible for reducing the risk of diabetes with the daily consumption of coffee have not yet been thoroughly investigated even though coffee is known to be a major source of dietary polyphenols.

Coffee is one of the most abundant supplies of dietary polyphenols. Fukushima et al. ${ }^{(9)}$ showed that coffee contributed to $50 \%$ of the daily consumption of polyphenols in Japanese individuals. The health benefits associated with the consumption of coffee may be linked to its content of polyphenols, which have various physiological functions ${ }^{(10)}$. Caffeic acid and its derivative, chlorogenic acid (a caffeic acid ester of quinic acid), have been identified as the most abundant polyphenols in coffee. A single cup of coffee contains 70-350 mg of chlorogenic acids ${ }^{(11)}$. We previously showed that dietary supplementation with coffee polyphenols reduced body fat and improved energy metabolism, and these findings were attributed to an increase in fat catabolism in the liver ${ }^{(12)}$. Our previous studies also demonstrated that the daily ingestion of chlorogenic acids increased fat catabolism in human subjects ${ }^{(13,14)}$. However, the relationship between the daily ingestion of chlorogenic acids and diabetes or blood glucose remains unknown.

Since the intestine is the first endocrine tissue involved in mediating the effects of coffee on whole-body glucose metabolism, our objective was to investigate the effects of dietary coffee polyphenols and chlorogenic acids on active GLP-1 secretion. The NCI-H716 cell line, which is derived from a poorly differentiated adenocarcinoma of the human caecum $^{(15)}$, has previously been shown to constitute a unique model of enteroendocrine cells that could be used to examine the cellular mechanism of GLP-1 secretion in human subjects ${ }^{(16)}$. We investigated the coffee polyphenol-induced release of active GLP-1(7-36) amide in NCI-H716 cells and C57BL/6J mice.

G protein-coupled receptor 119 (GPR119) was recently shown to mediate the secretion of GLP-1 by increasing cyclic AMP (cAMP) levels in several GLP-1-secreting cell models ${ }^{(17)}$. Since coffee polyphenols are known to stimulate active GLP-1 (7-36) amide and intracellular cAMP levels in vitro, we also determined whether coffee polyphenols could activate GPR119 signalling in vitro.

\section{Experimental methods}

\section{Materials}

All reagents for the experiments were from Sigma-Aldrich Japan K.K., unless otherwise stated. Roswell Park Memorial Institute (RPMI) 1640 medium, Dulbecco's modified Eagle's medium, antibiotic-antimycotic and fetal bovine serum (FBS) were purchased from Life Technologies.

Aprotinin solution was purchased from Wako Pure Chemical Industries, Ltd. A dipeptidyl peptidase IV (DPP IV) inhibitor was purchased from EMD Millipore Corporation.

\section{Coffee polyphenol extract}

Roasted coffee beans were infused with hot water, and the extract was applied to an aromatic-type adsorbent column (Sepabeads SP70; Mitsubishi Chemical). After washing the column with water, the polyphenol-rich fraction was eluted with $0.1 \% \mathrm{NaOH}$ and then neutralised with an ion-exchange resin to a weakly acidic $\mathrm{pH}$. The recovered polyphenol-rich fraction was concentrated to a solid content of $10 \%(\mathrm{w} / \mathrm{v})$ and spraydried to obtain coffee polyphenol extract (CPE). The composition of CPE was measured by HPLC. The total polyphenol content of CPE for in vitro or in vivo studies was 78.4 and $46.2 \%$, respectively. The composition of polyphenols was 8.5 \% 3-CQA (caffeolylquinic acid), 14.4\% 4-CQA, 24.4\% 5-CQA, 7.9 \% 3-FQA (feruloylquinic acid), $6.3 \%$ 4-FQA, $10 \cdot 0 \%$ 5-FQA, $10 \cdot 5 \%$ 3,4-di-CQA, $6.8 \%$ 3,5-di-CQA, and $11.0 \% 4,5$-di-CQA for the in vitro study. The composition of polyphenols for the in vivo study was $6.7 \% 3$-CQA, $7 \cdot 3 \%$ 4-CQA, 17.9 \% 5-CQA, $1.2 \%$ 3-FQA, $1.4 \%$ 4-FQA, $3.7 \%$ 5-FQA, $3.2 \% 3$,4-di-CQA, $1.9 \%$ 3,5-di-CQA, and $2 \cdot 8 \%$ 4,5-di-CQA. CPE contained no caffeine.

\section{Cell line and culture conditions}

Human NCI-H716 cells, developed in the laboratory of Dr Hernert Oie and colleagues, were obtained from the ATCC (American Type Cell Culture) under the permission of the National Institutes of Health $(\mathrm{NIH})$. These cells were grown in suspension at $37{ }^{\circ} \mathrm{C}$ with $5 \% \mathrm{CO}_{2}$. The culture medium was Roswell Park Memorial Institute (RPMI) 1640 supplemented with $10 \%$ FBS and 1/100 antibiotic-antimycotic. Endocrine differentiation was enhanced by growing cells in dishes coated with Matrigel (Becton Dickinson), as described previously $^{(18)}$.

\section{In vitro glucagon-like peptide-1 secretion study}

The GLP-1 secretion study was performed as described by Reimer $^{(19)}$ with slight modifications.

Briefly, $3 \mathrm{~d}$ before the study, $5 \times 10^{5}$ cells were seeded in twenty-four-well culture plates coated with Matrigel and containing high-glucose Dulbecco's modified Eagle's medium, $10 \%$ FBS and 1/100 antibiotic-antimycotic. On the day of the experiment, medium was replaced by Krebs-Ringer bicarbonate solution with or without test agents and $\mathrm{pH}$ was adjusted to $7 \cdot 2$. Fresh media were added and cells were incubated for $2 \mathrm{~h}$ at $37{ }^{\circ} \mathrm{C}$ with a $0-0 \cdot 1 \%$ (w/v) CPE solution. The number of cells in each well did not differ significantly even after cells had been treated with CPE for $2 \mathrm{~h}$. The effects of $2 \mu \mathrm{M}$-phorbol 12-myristate 13-acetate were examined as a positive control ${ }^{(16)}$. Supernatant fractions were collected with the addition of $10 \mu \mathrm{l}$ aprotinin solution $(60 \mathrm{kIU})$ and diprotin-A $(34 \mu \mathrm{g} / \mathrm{ml})$, and then frozen at $-80{ }^{\circ} \mathrm{C}$ for subsequent ELISA analyses. GLP-1 concentrations from the supernatant fractions were measured by Glucagon-Like Peptide-1 (Active) ELISA (Millipore Corporation). The basal level of GLP-1 secreted by the NCI-H716 cells for $2 \mathrm{~h}$ ranged 
between 131 and $392 \mathrm{pm}$ in Krebs-Ringer bicarbonate solution.

\section{Measurement of intracellular cyclic AMP levels}

GPR119 receptor EZCells (GPR119-transfected CHO-K1 (Chinese hamster ovary-K1) cells, Chan'Test Corp.), CHO-K1 cells and NCI-H716 cells were incubated for $20 \mathrm{~min}$ with 0-0.1\% CPE with $1 \mu \mathrm{M}$-isobutylmethylxanthine to avoid the degradation of cAMP, and cAMP levels were measured in the cell extract using a cAMP-Screen System (Applied Biosystems) according to the manufacturer's instructions.

\section{Animals}

Male C57BL/6J mice (12-13 weeks old; CLEA Japan Inc.) were housed at four or five mice per cage in a temperatureand relative humidity-controlled $\left(23 \pm 2 \cdot 0^{\circ} \mathrm{C}, 55 \pm 10 \%\right)$ room with a $12 \mathrm{~h}$ light $-12 \mathrm{~h}$ dark cycle with lights on at 07.00 hours. Mice were fed standard chow (CE-2; CLEA Japan, Inc.) consisting of $3.47 \mathrm{kcal}(14.52 \mathrm{~kJ}) / \mathrm{g}$ with $4.6 \%$ fat, $51.4 \%$ carbohydrate and $24.9 \%$ protein. Food and water were provided ad libitum. All animal experiments were conducted in the Experimental Animal Facility of Kao Tochigi Institute. The Animal Care Committee of Kao Corporation approved the present study.

\section{In vivo glucagon-like peptide-1 secretion study}

An oral glucose and fat load test was conducted as described previously ${ }^{(20)}$. Briefly, overnight-fasted mice were gastrically administered either $\mathrm{D}-(+)$-glucose or glyceryl trioleate (TO) (1 or $2 \mathrm{~g} / \mathrm{kg}$ body weight (BW) for each) alone, or glucose plus TO (1 or $2 \mathrm{~g} / \mathrm{kg}$ BW each). Lecithin (from egg yolk, $0.08 \mathrm{~g} / \mathrm{kg} \mathrm{BW}$ ) was included in all test solutions containing oil, and these were subsequently sonicated to obtain stable emulsions. We preliminarily confirmed that the minimal amount of lecithin did not affect blood glucose or insulin responses. In the first GLP-1 analysis, blood samples (approximately $300 \mu \mathrm{l}$ ) were collected via the portal vein for single blood time points (0, 10, 30, 60, 120 and $240 \mathrm{~min}$ ) under anaesthesia using micro blood collection tubes (Capiject; TERUMO) containing the DPP IV inhibitor (EMD Millipore Corporation) and aprotinin solution (Wako Pure Chemical Industries, Ltd). Our preliminary experiment failed to detect active GLP-1 in blood samples taken from the tail vein or orbital sinus; therefore, we collected blood samples via the portal vein for single blood time points $(0,10,30$, 60,120 and $240 \mathrm{~min}$ ) to estimate peak active GLP-1 levels. Overnight-fasted mice were divided into groups. One group was designated the ' 0 min' group, and blood samples were taken from the portal vein to determine fasting active GLP-1 levels. Blood samples were taken from the administered groups $10 \mathrm{~min}$ after gastric administration via the abdominal vein. Blood samples were collected into micro blood collection tubes containing the DPP IV inhibitor and aprotinin solution, and were maintained on ice. Within 30 min, blood samples were centrifuged and the collected plasma was stored at $-80^{\circ} \mathrm{C}$ until active GLP-1 ELISA analyses.

Overnight-fasted mice were gastrically administered water (control), D-(+)-glucose or starch (from waxy maize, $2 \mathrm{~g} / \mathrm{kg}$ $\mathrm{BW})$ plus TO $(1 \mathrm{~g} / \mathrm{kg} \mathrm{BW})$ with or without CPE $(0.6 \mathrm{~g} / \mathrm{kg}$ $\mathrm{BW}, 0.28 \mathrm{~g} / \mathrm{kg} \mathrm{BW}$ as polyphenols). Lecithin (from egg yolk, $0.08 \mathrm{~g} / \mathrm{kg} \mathrm{BW}$ ) was included in all test solutions containing oil, and these were subsequently sonicated to obtain stable emulsions. Blood samples (approximately $300 \mu \mathrm{l}$ ) were collected from the portal vein 10 or $30 \mathrm{~min}$ after the gastric administration because the time-course experiment estimated that active GLP-1 levels peaked at these time points. Blood samples were kept on ice until the preparation of plasma. After centrifugation, plasma was stored at $-80{ }^{\circ} \mathrm{C}$ until active GLP-1 ELISA analyses. Plasma GLP-1 was measured using a Glucagon-Like Peptide-1 (Active) ELISA kit (Millipore Corporation). Total glucose-dependent insulinotropic polypeptide (GIP) was measured using a Rat/Mouse GIP (total) ELISA kit (Millipore Corporation). Plasma insulin was measured using a Rat Insulin ELISA kit (Morinaga Institute of Biological Science, Inc.).

\section{Statistical analysis}

All values are presented as mean values with their standard errors. Unpaired Student's $t$ tests after a preliminary $F$ test of the homogeneity of within-group variance were used to compare values between the groups. When more than two groups were compared, statistical analysis was conducted with a one-way ANOVA followed by Fisher's protected least significant difference (PLSD) or Dunnett's post hoc tests (Statview for Windows version 5.0; SAS Institute Inc.). A P value of less than $0 \cdot 05$ was considered significant.

\section{Results}

\section{Active GLP-1(7-36) amide secretion from NCl-H716 cells}

Cells treated with phorbol 12-myristate 13-acetate for $2 \mathrm{~h}$ had significantly higher active GLP-1(7-36) concentrations in the media than those in the control. The glucose treatment also increased active GLP-1 secretion from cells in dose-dependent manner (Fig. 1(A)). Palmitic, oleic, linoleic or linolenic acid dose-dependently stimulated active GLP-1 secretion from cells (Fig. 1(B)). Active GLP-1 secretion was significantly greater with unsaturated fatty acids such as oleic, linoleic and linolenic acids than with palmitic acid. No significant differences were observed in active GLP-1(7-36) amide levels in the media between stearic acid and the control.

\section{Effects of coffee polyphenol extract on active GLP-1(7-36) amide secretion in vitro}

The treatment of NCI-H716 cells with CPE dose-dependently increased active GLP-1 concentrations in the media (Fig. 2). A $37 \%$ increase was observed in active GLP-1 secretion from these cells at a concentration of $0.1 \% \mathrm{CPE}$. 

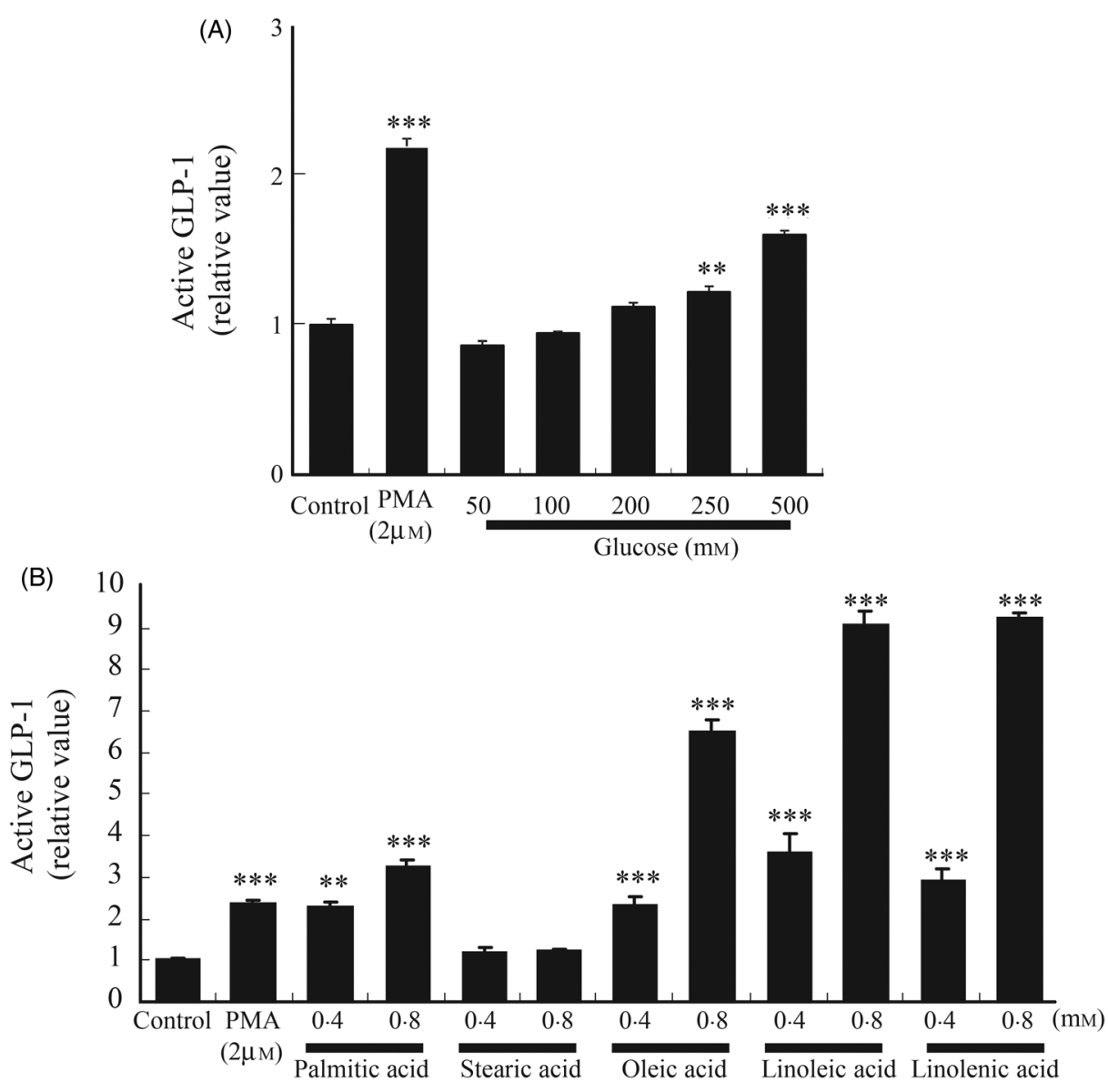

Fig. 1. Effects of glucose and fatty acids on active glucagon-like peptide-1 (GLP-1) secretion from NCl-H716 cells. Cells were incubated for $2 \mathrm{~h}$ with (A) glucose (50$500 \mathrm{~mm}$ ) or (B) fatty acids $(0.4$ or $0.8 \mathrm{~mm})$. Values are means ( $n-5$ per group), with standard errors represented by vertical bars. A one-way ANOVA followed by Dunnett's post hoc tests was used when comparing values between the control and experimental groups. Mean value was significantly different from that of the control group: ${ }^{* *} P<0.01,{ }^{* \star *} P<0.001$ (Dunnett's test). PMA, phorbol 12-myristate 13-acetate.

\section{Effects of coffee polyphenol extract on intracellular cyclic AMP} levels

The treatment of NCI-H716 cells with CPE increased cAMP levels in a dose-dependent manner (Fig. 3(A)). A significant increase was observed in intracellular cAMP levels at a concentration of $0.05 \%$ or more CPE. CPE increased intracellular cAMP in both GPR119-transfected cells or CHO-K1 control cells in a dose-dependent manner (Fig. 3(B)). No significant differences were observed in cAMP levels after the CPE treatment between GPR119-transfected and control cells.

\section{Active GLP-1(7-36) amide release in C57BL/6J mice}

Gastric administration of glucose increased active GLP-1(736) amide levels in the portal blood after $10 \mathrm{~min}$, and this was followed by a marked decrease in active GLP-1 levels at 30 min (Fig. 4(A)). The gastric administration of TO also increased active GLP-1 levels after $10 \mathrm{~min}$, and this was followed by a decrease to basal levels at $60 \mathrm{~min}$. A slight increase was noted in active GLP-1 levels in the portal blood $120 \mathrm{~min}$ after the administration of TO. Individually, glucose and TO increased the secretion of GLP-1 in a dose-dependent manner. Furthermore, the co-administration of glucose and TO additively increased peak GLP-1 levels (Fig. 4(B)).
Effects of coffee polyphenol extract on active GLP-1(7-36) amide release in vivo

CPE-administered mice had higher active GLP-1 levels in the portal blood at 10 and $30 \mathrm{~min}$ than those in the control mice (Fig. 5(C) and $(\mathrm{G})$ ). However, no significant difference was observed in GIP levels in the portal blood between the CPE-administered group and the control group (Fig. 5(D) and $(\mathrm{H})$ ). Neither glucose (Fig. 5(A) and (E)) nor insulin (Fig. 5(B) and (F)) levels were different between the control and CPE-administered groups.

When glucose was administered with CPE, active GLP-1 (Fig. 6(C)) and insulin (Fig. 6(B)) levels in the portal blood were slightly higher in $\mathrm{CPE}$-administered mice than in the control mice. Although blood glucose levels were similar between the CPE-administered and control groups at $10 \mathrm{~min}$, they declined more in the CPE-administered group, which resulted in significantly lower blood glucose levels in CPE-administered mice than in the control mice (Fig. 6(E)).

High-fat diet-fed C57BL/6J mice developed hyperglycaemia and impaired glucose tolerance. Therefore, the effects of coffee polyphenols given with the high-fat meal (starch $(2 \mathrm{~g} / \mathrm{kg} \mathrm{BW}$ ) plus trioleate $(1 \mathrm{~g} / \mathrm{kg} \mathrm{BW}), 33 \%$ (w/w) fat) on GLP-1 secretion were examined. When starch with TO was administered to mice, glucose levels were significantly increased in the portal 


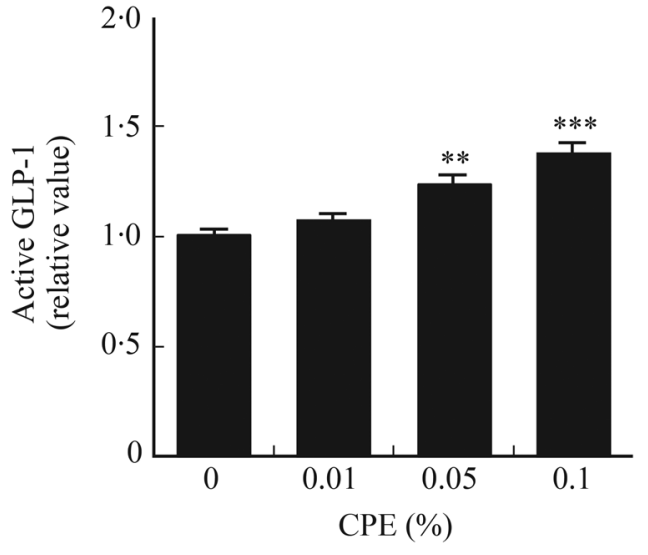

Fig. 2. Effects of coffee polyphenol extract (CPE) on active glucagon-like peptide-1 (GLP-1) secretion from NCl-H716 cells. Cells were incubated for $2 \mathrm{~h}$ with CPE $(0-0.1 \%)$. Values are means ( $n$ 14-16 per group), with standard errors represented by vertical bars. A one-way ANOVA followed by Dunnett's post hoc tests was used when comparing values between the control $(0 \%$ $\mathrm{CPE}$ ) and experimental groups. Mean value was significantly different from that of the control (0\% CPE) group: ${ }^{* *} P<0.01,{ }^{* * *} P<0.001$ (Dunnett's test).

blood at $10 \mathrm{~min}$ (Fig. 7(A)), followed by a decline in each blood variable at $30 \mathrm{~min}$ (Fig. 7(E)). The co-administration of $\mathrm{CPE}$ with starch plus TO significantly decreased peak blood glucose (Fig. 7(A)), GIP (Fig. 7(D)) and insulin (Fig. 7(B)) levels. No significant differences were observed in active GLP-1 levels in the portal blood between the CPE-administered and control groups at $10 \mathrm{~min}$ (Fig. 7(C)); however, these levels were significantly higher in the CPE-administered mice than in control mice at 30 min (Fig. $7(G)$ ).

\section{Discussion}

The present results support the hypothesis that polyphenols in coffee stimulate active GLP-1(7-36) amide secretion. The polyphenol extract isolated from coffee increased active GLP-1(7-36) amide release from NCI-H716 cells and in mice. To the best of our knowledge, this is the first study to demonstrate the direct stimulatory effects of coffee polyphenols on active GLP-1 secretion in vitro and in vivo.

Previous studies did not detect an increase in active GLP-1 levels in the peripheral blood following the ingestion of coffee polyphenols. Tunnicliffe et al. ${ }^{(21)}$ reported no significant changes in the postprandial secretion of GLP-1 by chlorogenic acid in either NCI-H716 cells or rats. Olthof et al. ${ }^{(22)}$ also did not find an overall effect of chlorogenic acid $(1 \mathrm{~g})$ ingested with glucose (75 g) on blood GLP-1 concentrations in overweight men. Several possible explanations could account for the increase observed in active GLP-1 levels after the ingestion of coffee polyphenols. One possibility is that we examined active GLP-1 levels in the portal blood. In preliminary experiments, active GLP-1(7-36) amide levels were lower in either peripheral or whole-blood samples than in portal blood samples even though blood was immediately treated with the DPP IV inhibitor. This difference in GLP-1 levels may be related to the susceptibility of secreted GLP-1 to inactivation by DPP $\mathrm{IV}^{(23)}$. Another possibility is that we examined the direct effects of coffee polyphenols on GLP-1 release. Previous
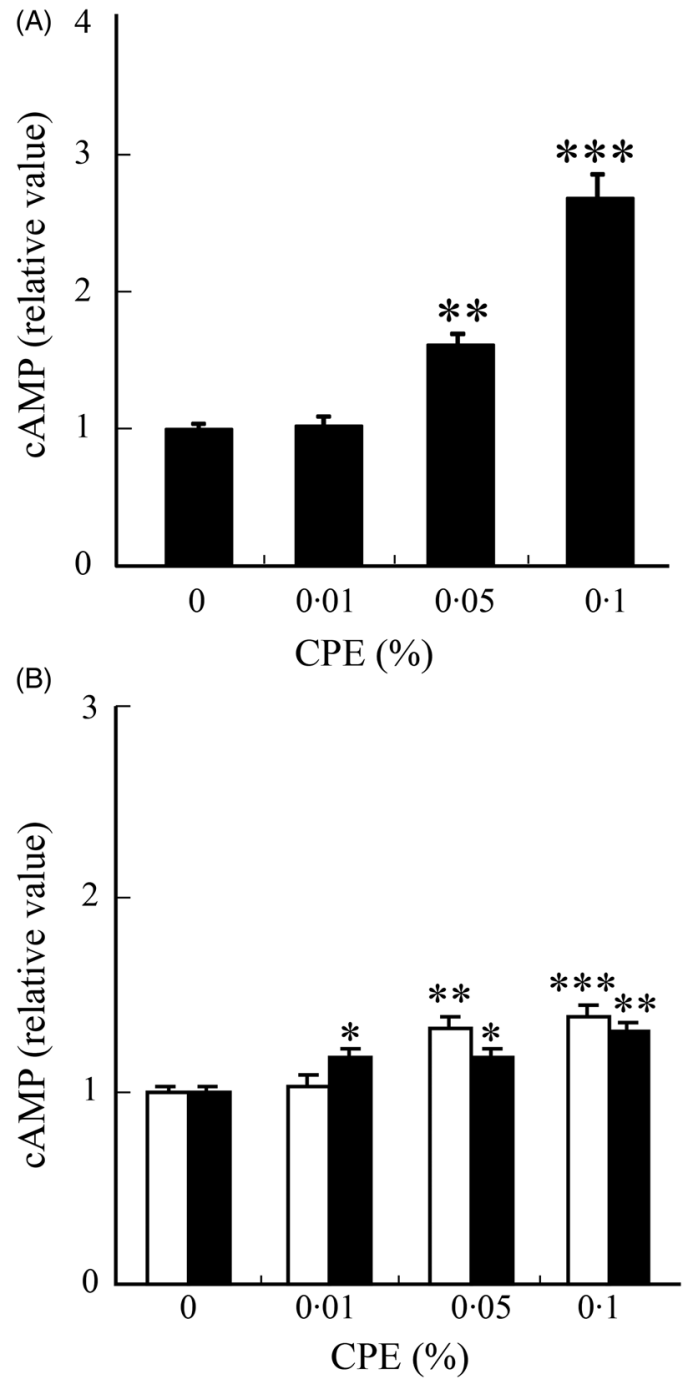

Fig. 3. Effects of coffee polyphenol extract (CPE) on intracellular cyclic AMP (cAMP) levels. (A) NCl-H716 cells were incubated for 20 min with CPE (0-0.1 $\%)$. (B) Chinese hamster ovary-K1 (CHO-K1) cells ( $\square$ ) and G protein-coupled receptor 119 (GPR119) receptor EZCells (ם) were incubated for 20 min with CPE (0-0.1\%). Values are means (n 3-4 per group), with standard errors represented by vertical bars. A one-way ANOVA followed by Dunnett's post hoc tests was used when comparing values between the control $(0 \% \mathrm{CPE})$ and experimental groups. Student's $t$ tests after a preliminary $F$ test of the homogeneity of within-group variance were used when comparing values between $0 \% \mathrm{CPE}$ and each concentration of CPE in GPR119 receptor EZCells (GPR119-transfected CHO-K1 cells). Mean value was significantly different from that of the control (0\% CPE) group: * $P<0.05,{ }^{\star *} P<0.01$, ${ }^{* * *}$ $P<0.001$ (Dunnett's test).

studies examined postprandial GLP-1 levels after the administration of chlorogenic acids with a meal or glucose. The stimulation of GLP-1 secretion by coffee polyphenols $(0.3 \mathrm{~g} / \mathrm{kg}$ $\mathrm{BW})$ was weaker than that by glucose $(2 \mathrm{~g} / \mathrm{kg} \mathrm{BW})$. A marked increase in the secretion of GLP-1 by a meal or the ingestion of glucose may obscure the stimulatory effects of coffee polyphenols. The present results demonstrated that coffee polyphenols, when ingested with glucose, increased GLP-1 and insulin levels in the portal blood, which appeared to result in a significant decrease in blood glucose levels thereafter. The results of the present study agree with those by Johnston et al. ${ }^{(24)}$, who reported higher blood GLP-1 levels after the consumption of decaffeinated coffee $(0 \cdot 35 \mathrm{~g}$ of chlorogenic 

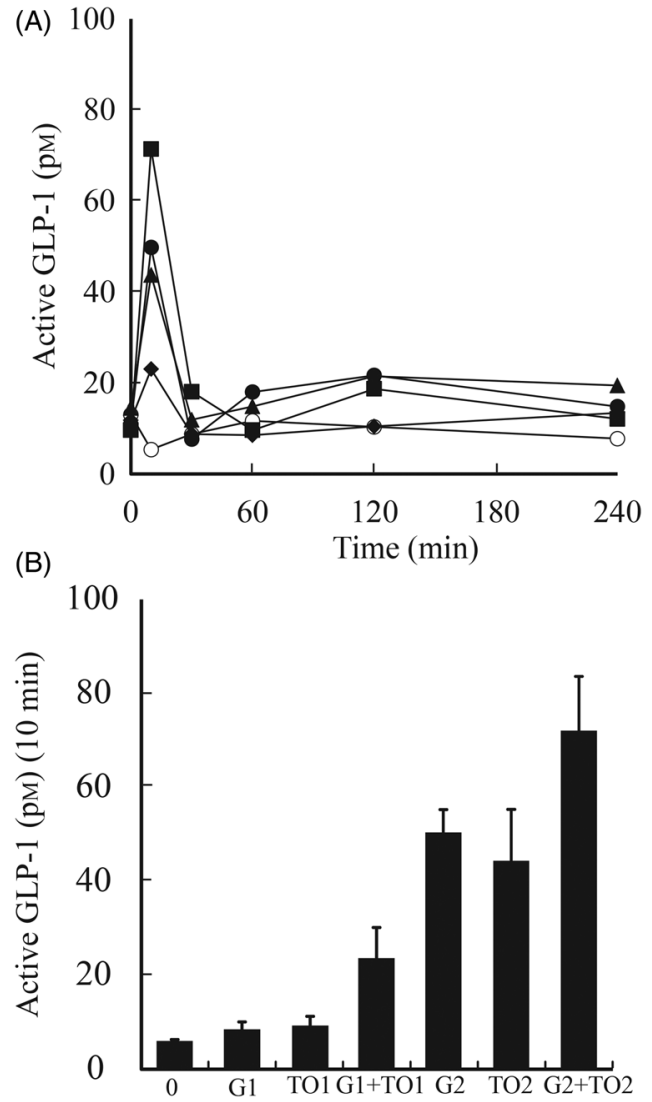

Fig. 4. Effects of glucose (G) and glyceryl trioleate (TO) on active glucagonlike peptide-1 (GLP-1) concentrations in the portal blood of C57BL/6J mice. (A) Time course of active GLP-1 and (B) active GLP-1 10 min after gastric administration. (- - ), 0 , Control; (- - ), G2, $2 \mathrm{~g} \mathrm{G} / \mathrm{kg}$ body weight (BW) (- $(-)$, TO2, $2 \mathrm{~g} \mathrm{TO} / \mathrm{kg} \mathrm{BW}$; (-), G1+TO1, $1 \mathrm{~g} \mathrm{G} / \mathrm{kg} \mathrm{BW}$ and $1 \mathrm{~g} \mathrm{TO} / \mathrm{kg}$ BW; (-), G2+TO2, $2 \mathrm{~g} \mathrm{G} / \mathrm{kg} \mathrm{BW}$ and $2 \mathrm{~g}$ TO/kg BW. Values are means ( $n 5$ per group), with standard errors represented by vertical bars.

acids) with glucose (25 g) than of control beverage in human subjects.

The present in vitro results demonstrated that glucose, fatty acids and coffee polyphenols directly stimulate active GLP-1 release from NCI-H716 cells in a dose-dependent manner. The present results also showed that glucose and TO similarly stimulate the secretion of GLP-1, and, when combined, they stimulate the secretion additively in mice. When administered with starch and fat, coffee polyphenols decreased postprandial blood responses from glucose, insulin and GIP. The present results are compatible with the findings of our previous study, which also showed that coffee polyphenols inhibit the activity of maltase ${ }^{(25)}$. Although the co-ingestion of coffee polyphenols with a meal decreased postprandial blood glucose, insulin and GIP responses, similar blood GLP-1 levels were retained as the control at $10 \mathrm{~min}$, and even higher GLP-1 levels at $30 \mathrm{~min}$ than control levels. The difference observed in the blood GLP-1 responses from glucose, insulin and GIP may have been due to the direct stimulatory effects of coffee polyphenols on the release of GLP-1.

Several GLP-1 secretagogues have so far been reported. Meat hydrolysate has been shown to stimulate the secretion of GLP-1 via the mitogen-activated protein kinase-dependent pathway ${ }^{(19)}$. Several amino acids are known to potentiate the
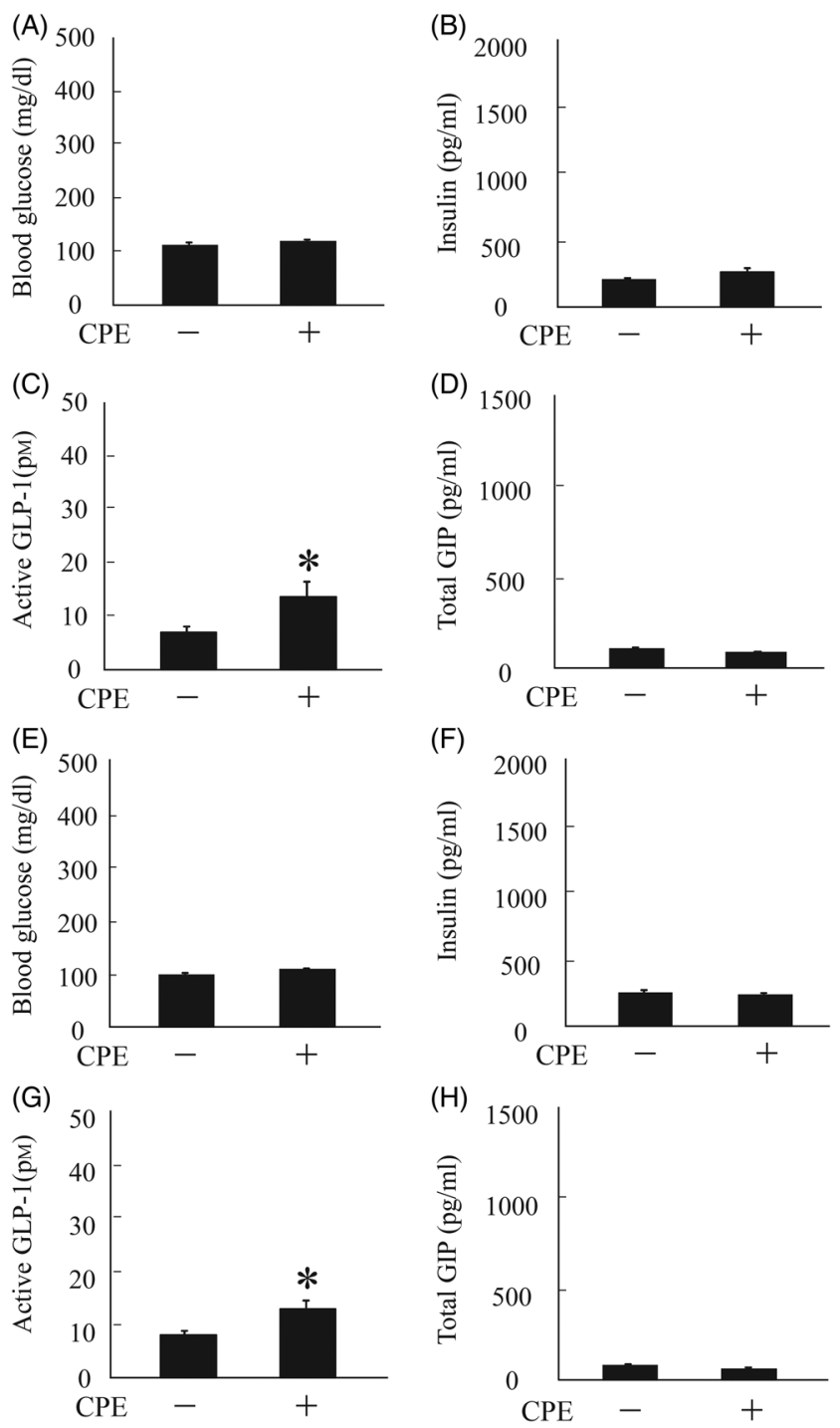

Fig. 5. Effects of coffee polyphenol extract (CPE) on glucose (A), insulin (B), active glucagon-like peptide-1 (GLP-1) (C) and glucose-dependent insulinotropic polypeptide (GIP) (D) concentrations in the portal blood of C57BL/6J mice 10 min after gastric administration. Effects of CPE on glucose $(E)$, insulin $(F)$, active GLP-1 (G) and GIP (H) concentrations in the portal blood of C57BL/6J mice 30 min after gastric administration. Values are means ( $n 6-8$ per group), with standard errors represented by vertical bars. Student's $t$ tests after a preliminary $F$ test of the homogeneity of within-group variance were used when comparing values between the groups. ${ }^{*}$ Mean value was significantly different from that of the control ( $0 \% \mathrm{CPE} ;-)$ group $(P<0.05$; Student's $t$ test). To convert glucose in $\mathrm{mg} / \mathrm{dl}$ to $\mathrm{mmol} / \mathrm{l}$, multiply by 0.0555 .

secretion of GLP-1 from GLUTag cells by increasing intracellular $\mathrm{Ca}^{2+}$ and cAMP levels ${ }^{(26)}$. Oleoylethanolamide was shown to stimulate the release of GLP-1 via the GPR119cAMP pathway ${ }^{(17)}$. The present results suggest that coffee polyphenols may trigger the secretion of GLP-1 from NCI-H716 cells via the cAMP-dependent pathway. In our preliminary experiment, GPR119 ligands such as oleoylethanolamide $(10 \mu \mathrm{M})$ and PSN632408 $(10 \mu \mathrm{M})$ increased cAMP levels in GPR119-transfected CHO-K1 cells by $2 \cdot 5$ - to $3 \cdot 5$-fold that of the control level (data not shown). In contrast, the increase in cAMP levels in the cells by CPE was only slight and equivalent to the increase in cAMP levels in CHO-K1 cells (GPR119-negative) (Fig. 3(B)). This result indicated that 
(A)
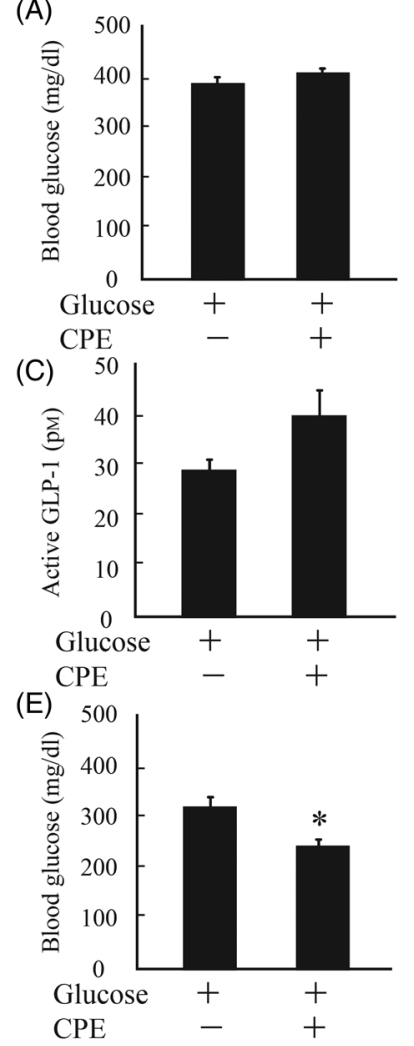

(G)

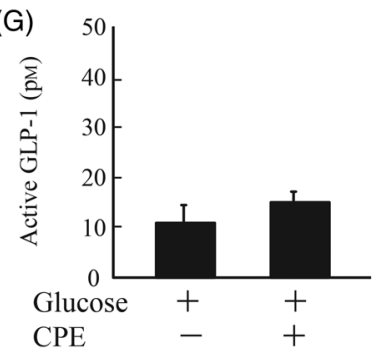

(B)

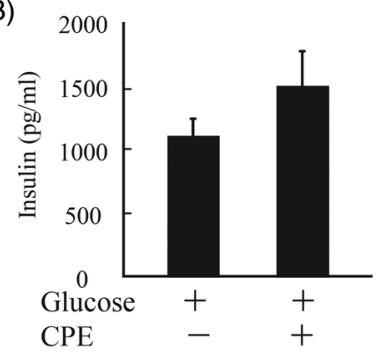

(D)

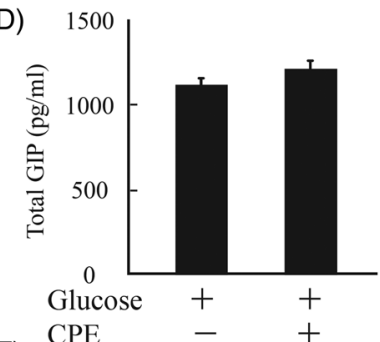

(F)

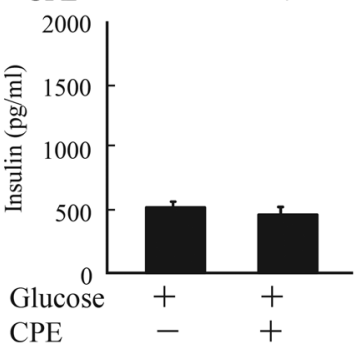

(H)

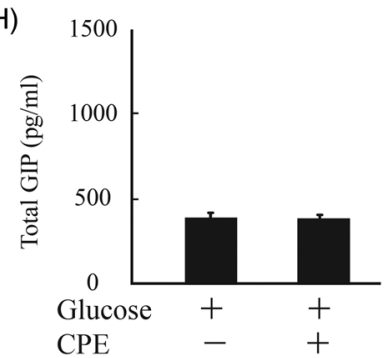

Fig. 6. Effects of coffee polyphenol extract (CPE) plus glucose on glucose (A), insulin (B), active glucagon-like peptide-1 (GLP-1) (C) and glucose-dependent insulinotropic polypeptide (GIP) (D) concentrations in the portal blood of C57BL/6J mice 10 min after gastric administration. Effects of CPE plus glucose on glucose $(E)$, insulin $(F)$, active GLP-1 $(G)$ and GIP $(H)$ concentrations in the portal blood of C57BL/6J mice $30 \mathrm{~min}$ after gastric administration. Values are means ( $n$ 7-8 per group), with standard errors represented by vertical bars. Student's $t$ tests after a preliminary $F$ test of the homogeneity of within-group variance were used when comparing values between the groups. * Mean value was significantly different from that of the control $(0 \% \mathrm{CPE} ;-)$ group ( $P<0.05$; Student's $t$ test). To convert glucose in $\mathrm{mg} / \mathrm{dl}$ to $\mathrm{mmol} / \mathrm{l}$, multiply by 0.0555 .

GPR119 was not responsible for the intracellular increase in cAMP by CPE. A limitation of the present study is that the molecules responsible for the secretion of GLP-1 triggered by coffee polyphenols remain unknown.

An increase in active GLP-1 levels may be beneficial by preventing the progression of diabetes. Despite the lack of a significant increase in active GLP-1 secretion or the insulinotropic activity of GLP-1, the results of the present study suggest that luminal coffee polyphenols may stimulate the secretion of active GLP-1, which consequently lowers postprandial hyperglycaemia. Active GLP-1 promotes cell growth $^{(27)}$ and inhibits cell apoptosis ${ }^{(28)}$ in pancreatic $\beta$-cells. While we have not yet demonstrated the prevention of
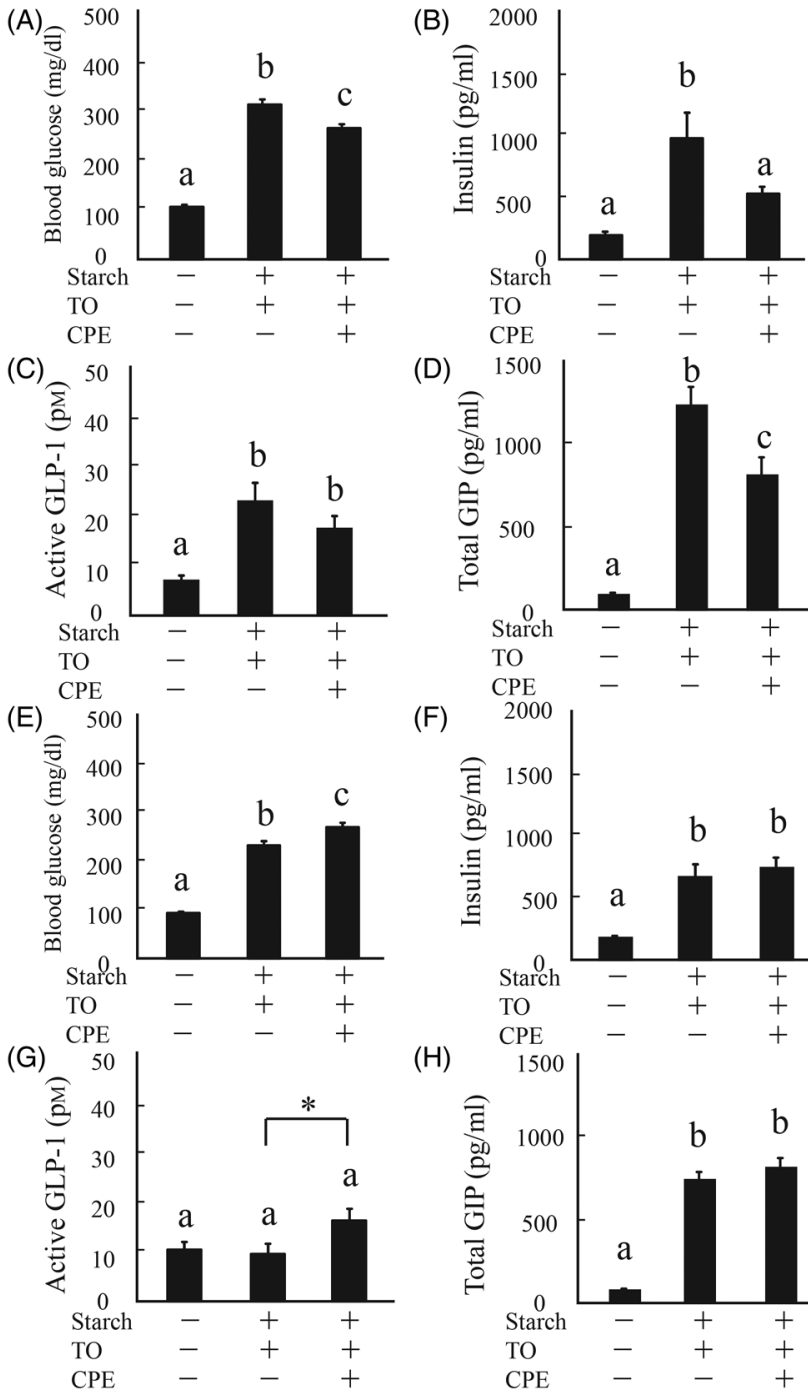

(D)

(F)

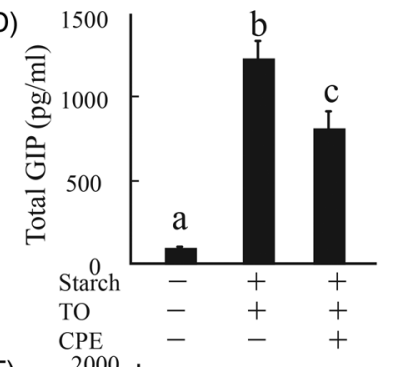

(H)

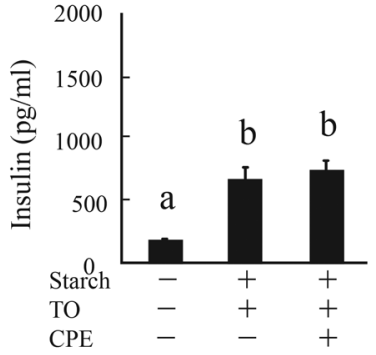

Fig. 7. Effects of coffee polyphenol extract (CPE) plus starch (from waxy maize) and glyceryl trioleate (TO) on glucose (A), insulin (B), active glucagonlike peptide-1 (GLP-1) (C) and glucose-dependent insulinotropic polypeptide (GIP) (D) concentrations in the portal blood of C57BL/6J mice $10 \mathrm{~min}$ after gastric administration. Effects of CPE plus starch (from waxy maize) and TO on glucose (A), insulin (B), GLP-1 (C) and GIP (D) concentrations in the portal blood of C57BL/6J mice $30 \mathrm{~min}$ after gastric administration. Values are means ( $n$ 9-11 per group), with standard errors represented by vertical bars. Statistical analysis was conducted using a one-way ANOVA followed by Fisher's protected least significant difference (PLSD) multiple comparison. a, b,c Mean values with unlike letters were significantly different $(P<0.05)$. * Mean values were significantly different $(P<0.05$; Student's $t$ test). To convert glucose in $\mathrm{mg} / \mathrm{dl}$ to $\mathrm{mmol} / \mathrm{l}$, multiply by 0.0555 .

diabetes by the chronic consumption of coffee polyphenols, the present results suggest that the reduced risk of type 2 diabetes due to the daily consumption of coffee may be explained, at least partly, by the intake of coffee polyphenols.

Although they do not detract from the significance of the present results, the limitations of the present study should be considered. The mechanism responsible for the stimulatory effects of coffee polyphenols including the $G$ protein-coupled receptor responsible and increase in intracellular cAMP levels remains unclear. Since GLP-1-secreting cells comprise less than $1 \%$ of the epithelial cell population in the small intestine, it is challenging to measure cAMP increases in enteroendocrine cells in vivo following the administration of coffee polyphenols. 
Further investigations, such as those examining the ligand activity of chlorogenic acid for $G$ protein-coupled receptors, will improve our understanding of the mechanism responsible for the stimulatory effects of coffee polyphenols on the release of GLP-1. Studies are currently being conducted to clarify whether coffee polyphenols stimulate postprandial active GLP-1 levels in humans, and also whether the daily consumption of coffee polyphenols prevents type 2 diabetes in animal models.

Although the postprandial secretion of the active GLP-1(736) amide was shown to be impaired in type 2 diabetic patients ${ }^{(29)}$, the insulinotropic activity of GLP-1, but not GIP, was preserved in patients with mild type 2 diabetes ${ }^{(30)}$. Exogenous GLP-1 improved fasting hyperglycaemia in type 2 diabetes $^{(31)}$. Taken together, active GLP-1 secretion by the daily consumption of coffee polyphenols may contribute to not only the prevention of, but also improvements in, type 2 diabetes. Therefore, the therapeutic value of the daily consumption of coffee polyphenols in combination with antidiabetic drugs such as DPP IV inhibitors needs to be examined in more detail.

\section{Conclusion}

In conclusion, the present study demonstrated that the ingestion of coffee polyphenols stimulates active GLP-1 secretion from enteroendocrine cells via the cAMP-dependent pathway. The present results suggest that polyphenols from coffee may serve as an exogenous physiological regulator of the secretion of incretin with an influence on blood glucose homeostasis. This may be important for the further development of GLP-1-based therapies for the treatment of diabetes. Therefore, more detailed studies on GLP-1 kinetics should be undertaken in relation to glucose homeostasis in larger animals and humans.

\section{Acknowledgements}

We thank Takuya Watanabe and Kiyoshi Kataoka for preparing the CPE.

The present study was supported financially by Kao Corporation.

A. S. managed the study, analysed the data and drafted the manuscript. Y. F., N. O. and A. S. were involved in the experiments as well as the collection and analysis of data. T. H. was involved in the conception of the study, management of research expenses and interpretation of data. All authors designed the study and critically revised the manuscript.

The authors declare that there are no conflicts of interest related to this paper.

\section{References}

1. International Diabetes Federation (2014) Diabetes Altas, 6th ed., 2014 update. http://www.idf.org/diabetesatlas (accessed November 2014)

2. Dorit SB, Olivia W, Synnott EL, et al. (2011) Glutamine reduces postprandial glycemia and augments the glucagon-like peptide-1 response in type 2 diabetes patients. J Nutr 141, 1233-1238.
3. Klatsky AL \& Armstrong MA (1992) Alcohol, smoking, coffee, and cirrhosis. Am J Epidemiol 136, 1248-1257.

4. La Vecchia C, Ferraroni M, Negri E, et al. (1989) Coffee consumption and digestive tract cancers. Cancer Res 49, 1049-1051.

5. Giovannucci E (1998) Meta-analysis of coffee consumption and risk of colorectal cancer. Am J Epidemiol 147, 1043-1052.

6. Sugiyama K, Kuriyama S, Akhter M, et al. (2006) Coffee consumption and mortality due to all causes, cardiovascular diseases, and cancer in Japanese women. Am J Clin Nutr 83, 1039-1046.

7. Van Dam RM \& Hu FB (2005) Coffee consumption and risk of type 2 diabetes: a systematic review. JAMA 294, 97-104.

8. Van Dam RM, Willett WC, Manson JE, et al. (2006) Coffee, caffeine, and risk of type 2 diabetes: a prospective cohort study in younger and middle-aged U.S. women. Diabetes Care 29, 398403.

9. Fukushima Y, Ohie T, Yonekawa Y, et al. (2009) Coffee and green tea as a large source of antioxidant polyphenols in the Japanese population. J Agric Food Chem 57, 1253-1259.

10. Fraga CG, Galleano M, Verstraeten SV, et al. (2010) Basic biochemical mechanisms behind the health benefits of polyphenols. Mol Aspects Med 31, 435-445.

11. Clifford MN (1999) Chlorogenic acids and other cinnamates: nature, occurrence and dietary burden. J Sci Food Agric 79, 362-372.

12. Murase T, Misawa K, Minegishi Y, et al. (2011) Coffee polyphenols suppress diet-induced body fat accumulation by downregulating SREBP-1c and related molecules in C57BL/6J mice. Am J Physiol Endocrinol Metab 300, E122-E133.

13. Ota N, Soga S, Murase T, et al. (2010) Consumption of coffee polyphenols increases fat utilization in humans. J Health Sci 56, 745-751.

14. Soga S, Ota N \& Shimotoyodome A (2013) Stimulation of postprandial fat utilization in healthy humans by daily consumption of chlorogenic acids. Biosci Biotech Biochem 77, 1633-1636.

15. Park JG, Oie HK, Sugarbaker PH, et al. (1987) Characteristics of cell lines established from human colorectal carcinoma. Cancer Res 47, 6710-6718.

16. Reimer RA, Darimont C, Gremlich S, et al. (2001) A human cellular model for studying the regulation of glucagon-like peptide- 1 secretion. Endocrinology 142, 4522-4528.

17. Lauffer LM, Iakoubov R \& Brubaker PL (2009) GPR119 is essential for oleoylethanolamide-induced glucagon-like peptide-1 secretion from the intestinal enteroendocrine L-cell. Diabetes 58, 1058-1066.

18. de Bruïne AP, Dinjens WN, van der Linden EP, et al. (1993) Extracellular matrix components induce endocrine differentiation in vitro in NCI-H716 cells. Am J Pathol 142, 773-782.

19. Reimer RA (2006) Meat hydrolysate and essential amino acidinduced glucagon-like peptide-1 secretion, in the human NCI-H716 enteroendocrine cell line, is regulated by extracellular signal-regulated kinase $1 / 2$ and p38 mitogen-activated protein kinases. J Endocrinol 191, 159-170.

20. Shimotoyodome A, Fukuoka D, Suzuki J, et al. (2009) Coingestion of acylglycerols differentially affects glucose-induced insulin secretion via glucose-dependent insulinotropic polypeptide in C57BL/ 6J mice. Endocrinology 150, 2118-2126.

21. Tunnicliffe JM, Eller LK, Reimer RA, et al. (2011) Chlorogenic acid differentially affects postprandial glucose and glucose-dependent insulinotropic polypeptide response in rats. Appl Physiol Nutr Metab 36, 650-659.

22. Olthof MR, Van Dijk AE, Deacon CF, et al. (2011) Acute effects of decaffeinated coffee and the major coffee components chlorogenic acid and trigonelline on incretin hormones. Nutr Metab 8, 10.

23. Hansen L, Deacon CF, Orskov C, et al. (1999) Glucagon-like peptide-1-(7-36) amide is transformed to glucagon-like peptide-1-(9-36) amide by dipeptidyl peptidase IV in the capillaries supplying the L cells of the porcine intestine. Endocrinology 140, $5356-5363$. 
24. Johnston KL, Clifford MN \& Morgan L (2003) Coffee acutely modifies gastrointestinal hormone secretion and glucose tolerance in humans: glycemic effects of chlorogenic acid and caffeine. Am J Clin Nutr 78, 728-733.

25. Murase T, Yokoi Y, Misawa K, et al. (2012) Coffee polyphenols modulate whole-body substrate oxidation and suppress postprandial hyperglycaemia, hyperinsulinaemia and hyperlipidaemia. $\mathrm{Br} J$ Nutr 28, 1757-1765.

26. Tolhurst G, Zheng Y, Parker HE, et al. (2011) Glutamine triggers and potentiates glucagon-like peptide- 1 secretion by raising cytosolic $\mathrm{Ca}^{2+}$ and cAMP. Endocrinology 152, 405-413.

27. Farilla L, Hongxiang H, Bertolotto C, et al. (2002) Glucagon-like peptide-1 promotes islet cell growth and inhibits apoptosis in Zucker diabetic rats. Endocrinology 143, 4397-4408.
28. Farilla L, Bulotta A, Hirshberg B, et al. (2003) Glucagon-like peptide 1 inhibits cell apoptosis and improves glucose responsiveness of freshly isolated human islets. Endocrinology 144, 5149-5158.

29. Vilsbøll T, Krarup T, Deacon CF, et al. (2001) Reduced postprandial concentrations of intact biologically active glucagon-like peptide 1 in type 2 diabetic patients. Diabetes 50, 609-613.

30. Nauck MA, Heimesaat MM, Orskov C, et al. (1993) Preserved incretin activity of glucagon-like peptide 1 [7-36 amide] but not of synthetic human gastric inhibitory polypeptide in patients with type-2 diabetes mellitus. J Clin Invest 91, 301-307.

31. Nauck MA, Kleine N, Orskov C, et al. (1993) Normalization of fasting hyperglycaemia by exogenous glucagon-like peptide 1 (7-36 amide) in type 2 (non-insulin-dependent) diabetic patients. Diabetologia 36, 741-744. 